\title{
Nanoscale
}

Cite this: Nanoscale, 2013, 5, 7403

Accepted 7th June 2013

DOI: $10.1039 / c 3 n r 01453 c$

www.rsc.org/nanoscale
Received 24th March 2013

\section{Localized surface plasmon-enhanced nanosensor platform using dual-responsive polymer nanocomposites $\uparrow$}

\author{
Jaesuk Choi, ${ }^{a}$ Min-Jae Choi, ${ }^{a}$ Jung-Keun Yoo, ${ }^{a}$ Woon Ik Park, ${ }^{a}$ Ju Ho Lee, ${ }^{\text {ab }}$ \\ Jeong Yong Lee ${ }^{\mathrm{ab}}$ and Yeon Sik Jung ${ }^{* a}$
}

\begin{abstract}
The fast and accurate identification of unknown liquids is important for the safety and security of human beings. Recently, sensors based on the localized surface plasmon resonance (LSPR) effect demonstrated an outstanding sensitivity in detecting chemical and biological species. In the present study, we suggest that a dual-responsive nanocomposite composed of two polymer brushes and two noble metal nanoparticles provides a significantly improved selectivity (improvement of a factor of 30 in figure-of-merit) for distinguishing diverse liquids compared to a single-responsive LSPR sensor. The dual-responsive LSPR sensor platform was realized by the synergic combinations of two hydrophobic and hydrophilic polymer brushes, which respond differently depending on the degree of interaction between the polymer brushes and the surrounding liquids. Moreover, the mixing ratio of two solvents can also be estimated with high accuracy using the dual-nanocomposite LSPR sensor, suggesting that this approach would be highly practical for in situ process monitoring systems that can instantly detect the change of solvent composition.
\end{abstract}

\section{Introduction}

Noble metal nanostructures have generated intense attention for the past decades due to their interesting optical properties. ${ }^{1,2}$ One of the important properties is collective oscillations of free electrons confined to the nanostructures, which are referred to as localized surface plasmon resonance (LSPR). Excitations of localized surface plasmons by incident light at certain frequencies result in the appearance of absorption bands, and their intensity and position are highly dependent on the size, size distribution, shape, composition, and spacing of the nanoparticles and the refractive index of the surrounding medium..$^{3-5}$ Numerous chemical and biological LSPR sensors have been developed through the capabilities of responding sensitively to the physical and chemical changes in surrounding media. ${ }^{6-8}$ In

\footnotetext{
${ }^{a}$ Department of Materials Science and Engineering, Korea Advanced Institute of Science and Technology (KAIST), 291 Daehak-ro, Yuseong-gu, Daejeon 305-701, Republic of Korea.E-mail: ysjung@kaist.ac.kr

${ }^{b}$ Center for Nanomaterials and Chemical Reactions, IBS, 291 Daehak-ro, Yuseong-gu, Daejeon 305-701, Republic of Korea

$\dagger$ Electronic supplementary information (ESI) available: Plots showing UV-Vis spectra of the dual-responsive nanocomposites under various types of surrounding media, the change of peak positions as a function of the solubility parameter, and classification of liquids depending on the type of peak shift. See DOI: $10.1039 / \mathrm{c} 3 \mathrm{nr} 01453 \mathrm{c}$

$\$$ Present address : Reliability Technology Research Center, Korea Electronics Technology Institute (KETI), 68 Yatap-dong, Bundang-gu, Seongnam 463-816, Republic of Korea.
}

most such cases, the key advantage of the LSPR effect is that the optical signals from nanosized noble metals can be easily measured via absorption spectra in the wavelength range visible to human eyes. Thus, it may enable the realization of a colorimetric sensor that can operate with the assistance of common mobile devices such as smart phones by measuring color changes using a conventional digital camera module. ${ }^{9}$

In the applications of detecting organic liquids, the change of optical signals in response to different refractive indices of surrounding media has been investigated in-depth., ${ }^{\mathbf{8} 10}$ While research has enhanced the sensitivity via the optimization of nanostructures, ${ }^{10}$ it should be pointed out that the similarity of the refractive indices among many organic liquids can lead to poor selectivity. On the other hand, LSPR sensors based on the manipulation of interparticle distances of plasmonic nanoparticles (NPs) have been demonstrated. ${ }^{11,12}$ This approach mainly exploits the interparticle coupling effect, which is controlled by the swelling-deswelling behavior of responsive polymer brushes anchored on substrates. ${ }^{13}$ The real-time monitoring of swellingdeswelling behaviors is possible for stimuli-responsive polymer brushes. ${ }^{11,12}$ Moreover, the swelling ratio along the thickness direction is highly dependent on the surrounding environment, ${ }^{14,15}$ which is practical for the simple, convenient measurement of the degree of polymer-solvent interaction.

The detection of various types of organic liquids using LSPR nanostructures utilizes different peak positions in absorption spectra because the stretching of polymer chains in good solvents leads to an increase in the interparticle distances 
between NPs and induces a shift of the peaks toward shorter wavelengths. However, despite the apparent simplicity of fabricating plasmonic nanostructures and their outstanding convenience when used, the significant overlaps of the LSPR absorption bands among organic liquids make it difficult to distinguish them with high accuracy. For instance, the interactions between a certain polymer chain and several organic liquids can result in similar degrees of swelling and, consequently, comparable interparticle distances and absorption peak shifts for plasmonic NPs. Alternatively, even if there is a significant difference in the degree of polymer-liquid interactions, the degree of peak shifts can be reduced or even cancelled out depending on the refractive indices of the surrounding liquids, as we show in examples in this study. In addition, the variation ranges of the polymer swelling behavior and nanoparticle sizes are other factors that can result in ambiguity in the precise identification of liquid samples.

Here, we report a dual-responsive plasmonic nanocomposite sensor that exhibits two independent LSPR signals and show that it significantly improves the detection selectivity for the identification of unknown liquids. The sensor device was fabricated by adopting two plasmonic nanocomposites with distinct levels of responsiveness toward the surrounding liquids and with separate locations of their LSPR absorption peaks. By placing the different nanocomposite films on two opposite sides of a transparent substrate, a single absorption measurement can generate two peak positions for a liquid and locate it at a single spot on a twodimensional (2D) map constructed with a database of diverse liquids. We show that this $2 \mathrm{D}$ identification scheme ${ }^{\mathbf{1 6 , 1 7}}$ can reduce the degree of the overlap of absorption peaks among different liquids considerably and can differentiate them with a greatly enhanced precision. Moreover, we report the clear changes in the peak positions depending on the mixing ratio for two known liquids, providing the additional capability to estimate the composition of liquid mixtures accurately.

\section{Results and discussions}

Thin films which are responsive to neighboring liquids can be fabricated based on nanocomposites properly composed of polymer brushes and noble metal nanoparticles. First, to achieve a high degree of responsiveness to various types of organic liquids with different solubility parameters, we chose a hydrophobic polymer, polystyrene (PS), and a relatively hydrophilic polymer, poly(2-vinylpyridine) (P2VP). PS can swell more easily by hydrophobic solvents with smaller solubility parameters, while P2VP is more responsive to hydrophilic solvents with larger solubility parameters, which provided dual responsiveness toward surrounding liquids. Also, two noble metals having absorption peaks at considerably different wavelength ranges should be selected. Thus, we selected pairs of silver nanoparticles (Ag NPs) anchored onto P2VP brushes and gold nanoparticles (Au NPs) tethered onto PS brushes.

Fig. 1a shows the schematic procedure for the fabrication of two LSPR nanocomposites on two opposite sides of a glass substrate. First, hydroxyl-terminated P2VP homopolymers were spin-cast onto one side of a glass substrate and were then (a)

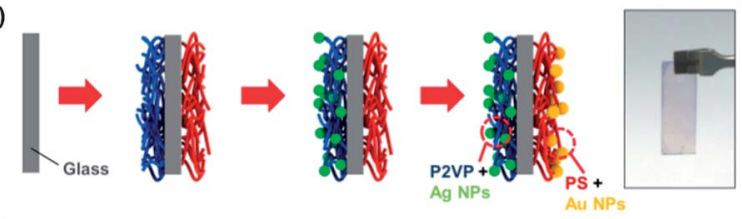

(b)
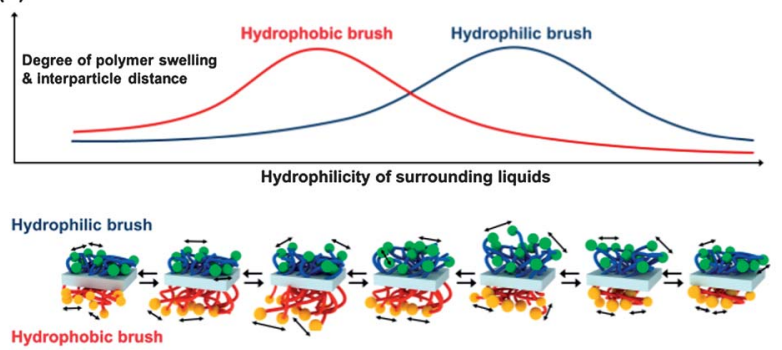

(c)

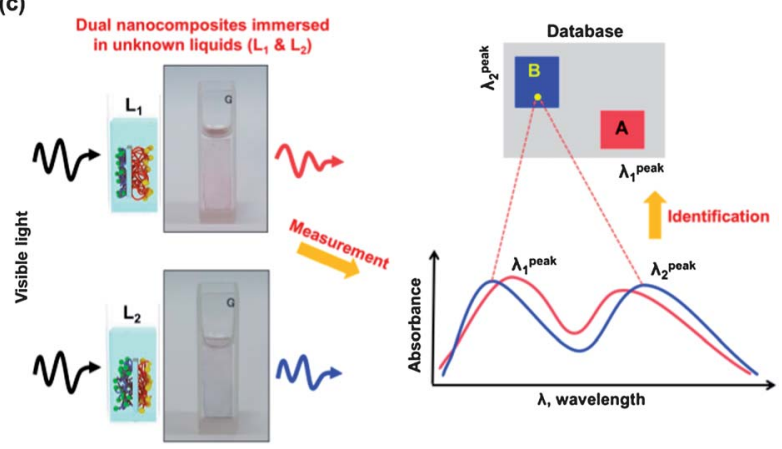

Fig. 1 (a) Process scheme for the fabrication of a dual-responsive plasmonic sensor. First, two polymer brushes are grafted on a glass substrate. This is followed by the formation of metal nanoparticles on the brushes. As a result, two types of nanocomposites (P2VP/Ag NP and PS/Au NP) are formed on the opposite faces of a glass substrate. (b) Schematic of swelling and deswelling behaviors of the two nanocomposites using different liquids. The degree of polymer swelling and the average distance among the attached nanoparticles are maximized when the solubility parameters of the polymers and surrounding liquids are closely matched. (c) Procedure to identify unknown liquids using the dual-responsive nanocomposite sensor. Because the two nanocomposites respond differently depending on the degree of interaction between the polymers and the liquids, two specific peak positions ( $\lambda_{1}^{\text {peak }}$ and $\lambda_{2}^{\text {peak }}$ ) can be used for the identification of liquids in a wide range with high selectivity.

thermally treated for permanent attachment as a polymer brush. Subsequently, a poly(glycidyl methacrylate) (PGMA) anchoring layer was deposited on the other side of the glass substrate for the formation of functionalized PS brushes. ${ }^{18}$ On the PGMA thin films, bi-functionalized ( $\alpha$-hydroxy, $\omega$-carboxy terminated) PS homopolymers were spin-coated and thermally annealed for chemical anchoring on the glass substrate. The epoxy groups of PGMA have strong reactivity with carboxyl groups; ${ }^{19}$ thus, the $\mathrm{COOH}$-terminated end groups of the bifunctionalized PS homopolymers are grafted chemically to the PGMA layer, leading to OH-terminated PS brushes on a glass substrate. All of the unreacted polymers were washed away with solvents (pyridine for P2VP and toluene for PS).

$\mathrm{Ag}$ NPs were then immobilized onto the P2VP brush layer. As the pyridine groups in P2VP chains have strong affinity toward metal ions, ${ }^{20} \mathrm{Ag}$ ions were absorbed on P2VP chains by soaking the brush-coated substrate in an $\mathrm{AgNO}_{3}$ aqueous solution. We 
then obtained Ag NPs in P2VP chains by reducing Ag ions chemically using an aqueous solution of $\mathrm{NaBH}_{4}$. The surface morphology of the obtained P2VP nanocomposites embedded with Ag NPs (P2VP/Ag NP) was characterized by atomic force microscopy (AFM). Fig. 2a and b respectively show surface topographic images of the samples before and after the attachment of the $\mathrm{Ag}$ NPs. The surface roughness of the P2VP/Ag NP sample (Fig. 2b) was estimated to be slightly higher (root-mean-square roughness, $\left.\sigma_{\mathrm{rms}}=0.64 \mathrm{~nm}\right)$ than that $\left(\sigma_{\mathrm{rms}}=0.23 \mathrm{~nm}\right)$ of the bare P2VP brushes (Fig. 2a). However, because most Ag NPs were formed inside the P2VP brush, only a small fraction of Ag NPs can be imaged by AFM, as shown in Fig. 2b. However, transmission electron microscopy (TEM) images, as shown in Fig. 2e, confirm the dense dispersion of Ag NPs with a mean diameter of $5 \mathrm{~nm}$ below the surface of the P2VP brushes.

For the fabrication of the other LSPR nanocomposite based on Au NPs and PS brushes, NP synthesis and attachment steps were separated. After the synthesis of $\mathrm{Au} \mathrm{NPs},{ }^{21,22}$ they were attached on the opposite side of the substrate with end-functionalized PS brushes. The core Au NPs were surrounded by surfactant molecules (11-mercaptoundecanoic acid) with free carboxylic acid groups, ${ }^{22}$ leading to the capability of hydrogen bonding with the OH-groups on PS brushes. In order to avoid linkage formation between Au NPs and the pyridine groups of the P2VP chains, the Au NP solution was drop-cast only onto the (a)

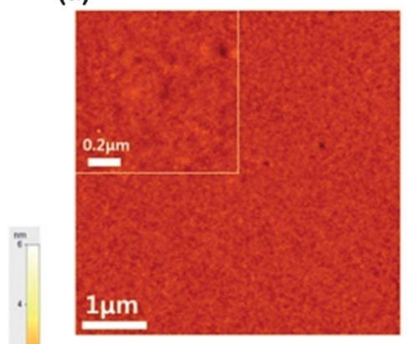

(c)

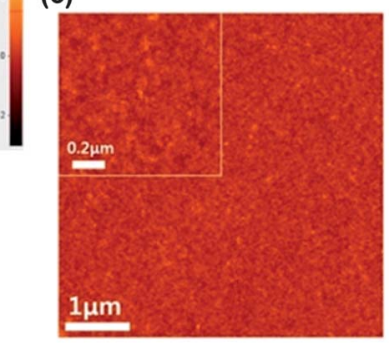

(e)

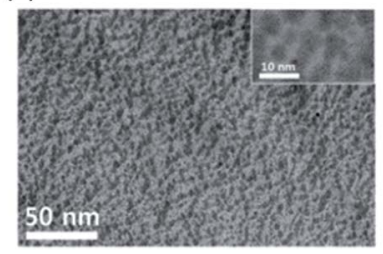

(b)

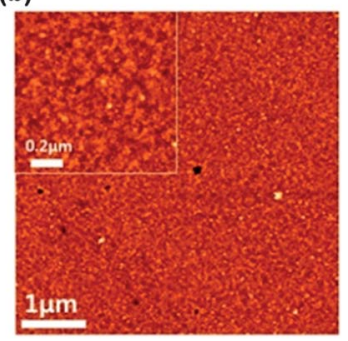

(d)

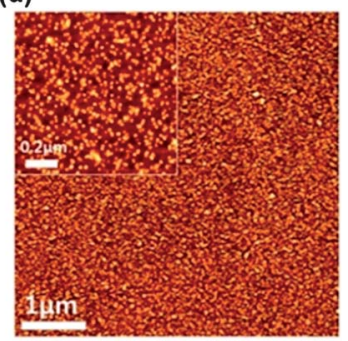

(f)

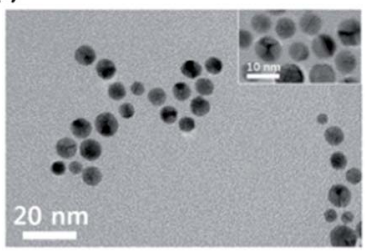

Fig. 2 AFM and TEM images: (a-d) AFM surface topographic images ( $5 \mu \mathrm{m} \times$ $5 \mu \mathrm{m}$ ) (a) before and (b) after the formation of Ag nanoparticles on P2VP brushes using a chemical reduction process, and (c) before and (d) after the attachment of Au nanoparticles onto the PS brushes. TEM images of (e) Ag nanoparticles and ( $f$ ) Au nanoparticles. The insets show enlarged images. substrate face with PS brushes. Fig. $2 c$ and d show AFM surface images before and after, respectively, the fixation of Au NPs onto the PS brushes. In contrast to the case of P2VP/Ag NP, the dense array of Au NPs is clearly visible in the AFM images because they exist mainly on the surface of the polymer brush, as the Au NP surface strongly interacts with the OH-groups on the brushes. Therefore, the PS/Au NP surface has higher values of $\sigma_{\mathrm{rms}}(=1.51 \mathrm{~nm})$ compared to that $(=0.64 \mathrm{~nm})$ of P2VP/Ag NP. The TEM images in Fig. 2f reveal that the Au NPs have an average size of $8 \mathrm{~nm}$.

The generation of double absorption peaks of the dualnanocomposite sensor is schematically demonstrated in Fig. 1b and c. The individual LSPR nanocomposites formed on the opposite sides respond differently depending on the degree of interactions between the polymer brushes and surrounding liquids. As schematically described in Fig. 1b, the degree of brush swelling along the vertical direction and inter-nanoparticle distances are maximized when their solubility parameters are closely matched. From the absorption spectra of the semi-transparent nanocomposite sensor immersed in a liquid, two data points $\left(\lambda_{1}^{\text {peak }}\right.$ and $\left.\lambda_{2}^{\text {peak }}\right)$ are determined by finding the wavelengths with the maximum absorption. Because this sensor platform can provide double information via single measurement, its analysis throughput would be higher than the case of multiple measurements for individual sensors.

As shown in Fig. 1c, the peak shifts of absorption spectra can result in a color change of the dual nanocomposite, suggesting that the nanocomposite can be promising for a colorimetric sensor with much superior portability. Fig. 3a shows a comparison of the UV-Vis absorption spectra from pure brushes (without NPs) and from nanocomposites under different environments (air, toluene, and acetic acid). No noticeable peaks in the visible range are shown for the bare brush, whereas the NP incorporation in the polymers leads to two peaks at $433 \mathrm{~nm}$ (first peak, $\lambda_{1}^{\text {peak }}$ ) and at $567 \mathrm{~nm}$ (second peak, $\lambda_{2}^{\text {peak }}$ ), which originate from the $\mathrm{Ag}$ and $\mathrm{Au}$ NPs, respectively. ${ }^{12,23}$ The immersion of the nanocomposite sensor in toluene resulted in a clear blue shift of the second peak (PS/Au NP), whereas the first peak (P2VP/Ag NP) did not respond significantly. This can be understood by considering the dominant swelling of the PS (solubility parameter, $\delta=18.5 \mathrm{MPa}^{1 / 2}$ ) and the marginal stretching of the P2VP $\left(\delta=20.6 \mathrm{MPa}^{1 / 2}\right)$ chains in toluene $\left(\delta=18.3 \mathrm{MPa}^{1 / 2}\right) .^{24,25}$ On the other hand, immersion in acetic acid $\left(\delta=21.4 \mathrm{MPa}^{1 / 2}\right)^{24}$ clearly decreased $\lambda_{1}^{\text {peak }}$, while $\lambda_{2}^{\text {peak }}$ remained almost constant. Fig. S1a shows a graph of the UV-Vis spectra taken after dipping the sensor in 13 different solvents (ESI $\dagger$ ). A closer examination of the data reveals that two absorption bands in various liquids have different combinations of peak positions ( $\lambda_{1}^{\text {peak }}$ and $\left.\lambda_{2}^{\text {peak }}\right)$ which are shifted positively and negatively from those of the reference dry state. The negative peak shifts are mainly due to the swelling effect of the polymer brushes caused by the solvents compared to the influence of the refractive indices of the surrounding media. This is supported by the plots in Fig. S2a and b (ESI $\dagger$ ), where the degree of blue shift was higher for the solvent with a solubility parameter similar to that of the polymer brushes, suggesting that the dominant factor for determining the peak positions is the degree of interaction between the polymer brushes and 
(a)

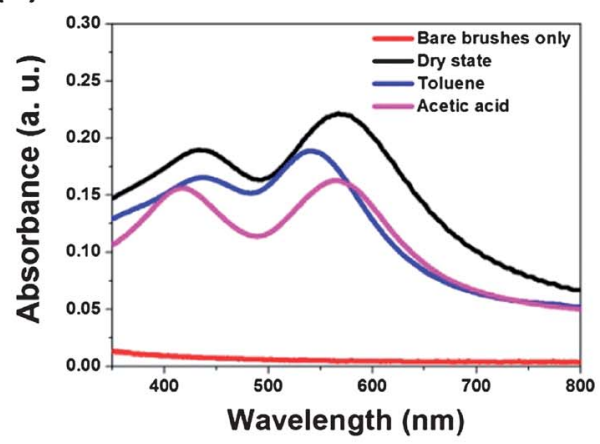

(b)

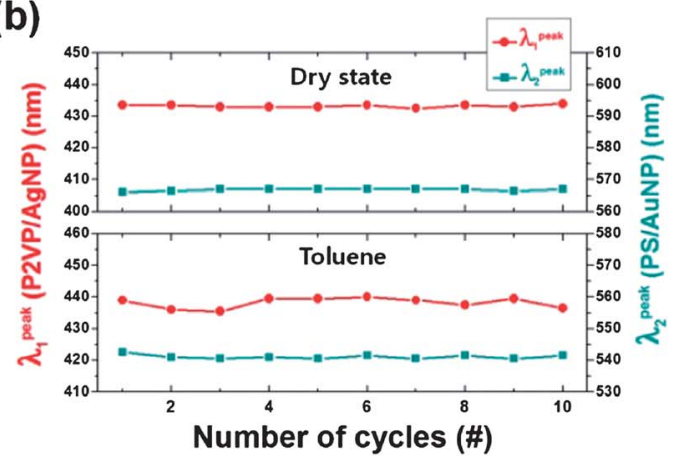

(c)

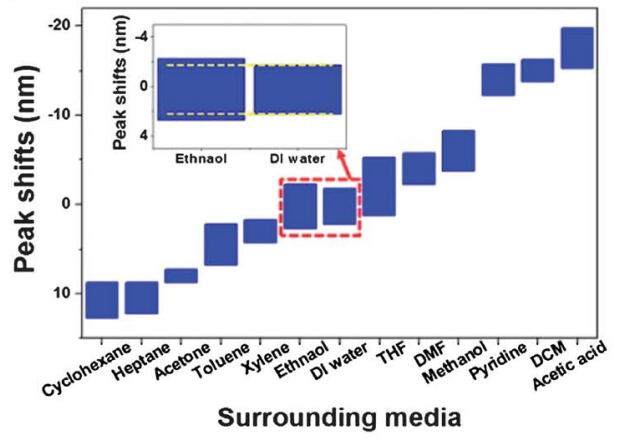

(d)

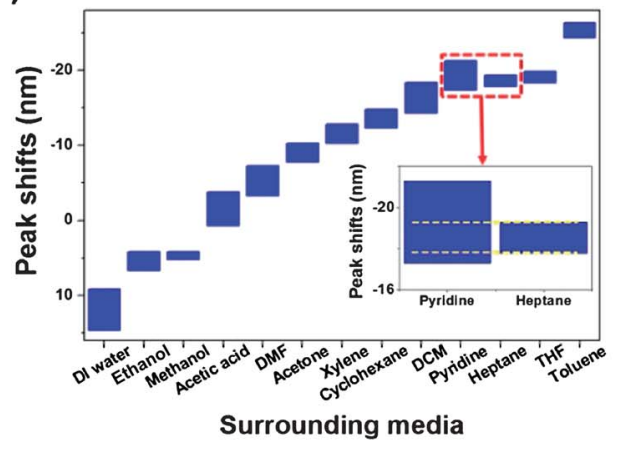

Fig. 3 (a) UV-Vis spectra of pure brushes and nanocomposites in different environments. The dual nanocomposite shows two separate absorption bands, and their peak wavelengths ( $\lambda_{1}^{\text {peak }}$ and $\lambda_{2}^{\text {peak }}$ ) significantly change depending on the surrounding media. (b) The repetitive measurement data of peak positions $\left(\lambda_{1}^{\text {peak }}\right.$ and $\lambda_{2}^{\text {peak }}$ ) for the dry state and in toluene. (c and $\left.d\right)$ The ranges of $\lambda^{\text {peak }}$ shifts for different liquids (reference $=$ dry state) (c) P2VP/Ag NP ( $\lambda_{1}^{\text {peak }}$ ) and (d) PS/Au NP $\left(\lambda 2^{\text {eak }}\right)$. The data were collected 10 times in each case. surrounding solvents. In contrast, if the degree of swelling in the polymer brushes is insignificant, a positive shift in proportion to the refractive index of a liquid is observed. ${ }^{26}$

The repeatability measurement results for the two individual absorption peak positions are shown in Fig. 3b. Although the occurrence positions for $\lambda^{\text {peak }}$ were reproducible as the number of cycles increased, some degree of variation in the peak positions was noted. For example, the measured values of $\lambda_{1}^{\text {peak }}$ and $\lambda_{2}^{\text {peak }}$ for toluene were 435.5-440 $\mathrm{nm}$ and 540.5-542.5 nm, respectively. Thus, the ranges (the differences between the maximum $\lambda^{\text {peak }}$ and the minimum $\lambda^{\text {peak }}$ ) were estimated to be $4.5 \mathrm{~nm}$ (for $\lambda_{1}^{\text {peak}}$ ) and $2 \mathrm{~nm}$ (for $\lambda_{2}^{\text {peak }}$ ), respectively. All of the peak shift ranges (reference $=$ dry state) of the 13 solvents are shown in Fig. 3c and d. The measured peak ranges for the different solvents present significant overlaps, which stem from the combined effects of polymer chain stretching, the refractive indices of the liquids, and the measurement accuracy, as mentioned above. For instance, the inset in Fig. 3c shows that the overlap range between ethanol and deionized water is $4 \mathrm{~nm}$, which is significant considering that the variations of $\lambda_{1}^{\text {peak }}$ (absorption peak from P2VP/Ag NP) are $5 \mathrm{~nm}$ and $4 \mathrm{~nm}$, respectively. This type of mutual overlap was observed for 18 pairs among the 13 solvents. Also for the $\lambda_{2}^{\text {peak }}$ values (corresponding to PS/Au NP), 11 cases of significant overlap occurred, as shown in Fig. 3d. We can more quantitatively estimate the total degree of overlap (DOO) using the following expression:

$$
\frac{\sum_{i=1, j=1, i \neq j}^{n, n}\left(\frac{\operatorname{overlap}(i, j)}{\operatorname{range}(i)+\operatorname{range}(j)}\right)}{{ }_{n} C_{2}}
$$

here, overlap $(i, j)$ is the mutual overlap range of $\lambda^{\text {peak }}$ between two solvents $\left(i\right.$ and $j$ ), range $(i)$ is the $\lambda^{\text {peak }}$ variation range for the solvent $(i)$, and ${ }_{n} C_{2}$ is the total number of pairs among the solvents. When DOO is 0 , there is no overlap range for any solvent, and when DOO is 1 , all solvents have the same ranges, indicating that a lower DOO value represents better selectivity. For P2VP/Ag NP, DOO is calculated to be 0.0857 for the 13 solvents investigated in this study, while the DOO value of PS/Au NP is 0.0414 .

We now demonstrate how the dual-responsive nanocomposite sensor can effectively distinguish different liquids. As shown in the two-dimensional map of $\lambda_{1}^{\text {peak }}$ and $\lambda_{2}^{\text {peak }}$ for the 13 solvents (Fig. 4a), except for pyridine and dichloromethane (DCM), all of the other solvents occupy different areas without mutual overlapping in the map. For example, ethanol and deionized water, which cannot clearly be distinguished by the $\mathrm{P} 2 \mathrm{VP} / \mathrm{Ag}$ NP sensor, can be differentiated using the dual nanocomposites. As a result, the number of pairs partially sharing their $\lambda^{\text {peak }}$ range decreased to 1 for all 13 solvents. The calculation of DOO extended to the two-dimensional map, where the variation ranges of $\lambda_{1}^{\text {peak }}$ and $\lambda_{2}^{\text {peak }}$ are represented as rectangles, leads to a value of 0.0013 , which is significantly smaller than 0.0857 and 0.0414 as estimated for the one-dimensional case. (Fig. 4b) Even for the single pair of pyridine and DCM, a substantial reduction of DOO from 0.8333 and 0.2500 to 0.1020 was achieved, suggesting a better differentiation accuracy compared to the single nanocomposite sensors. 
(a)

Considering the significant improvement of selectivity due to the use of the dual-responsive LSPR sensor, a triple-responsive sensor constructed by adding one additional nanocomposite to the dual-responsive sensor can be even more advantageous. To extend the detection range, the third nanocomposite may need to be composed of a different noble metal (e.g., Pt or Pd) with an absorption peak in a different wavelength range and a polymer brush which is more hydrophilic (e.g., polyethylene oxide) compared to P2VP or more hydrophobic (e.g., polyethylene) compared to PS. Further improved detection accuracy and a wider detection range are expected from such multipleresponsive nanocomposites.

Depending on the types of responses by the nanocomposite sensor, the 13 liquids can be classified into four groups, as represented by rectangular areas denoted as A, B, C, and D, respectively (Fig. S3, ESI $\dagger$ ). The liquids in region $\mathrm{B}$ do not swell either P2VP or PS; thus, both $\lambda_{1}^{\text {peak }}$ and $\lambda_{2}^{\text {peak }}$ show shifts toward larger wavelengths, which are induced by the higher refractive indices of the liquids compared to air. On the other hand, P2VP and PS are significantly swollen by the liquids belonging to region $\mathrm{C}$, leading to blue shifts. The other liquids categorized in regions A and D can swell P2VP or PS, respectively, without interacting with the other polymer significantly.
We also demonstrate that this LSPR sensor can be used for estimating the composition of two known liquids that are mixed together. First, we prepared five solvents of pure toluene and mixed solutions of toluene and dimethylformamide (DMF) at mixing ratios of $4: 1,1: 1$, and $1: 4$, and pure DMF. With an increase in the fraction of DMF, the curve follows an L-shaped graph, as shown in Fig. 5a. The preferential swelling of PS by toluene, which induced a blue shift of $\lambda_{2}^{\text {peak }}$ and a red shift of $\lambda_{1}^{\text {peak }}$, located pure toluene at the lower-right position on the graph. However, the gradual addition of DMF increased the degree of P2VP swelling, and consequently, the magnitude of the blue shift of $\lambda_{1}^{\text {peak }}$ increased. The abrupt transition in the positions of $\lambda_{1}^{\text {peak }}$ and $\lambda_{2}^{\text {peak }}$ originated possibly from the strong preferential solvation of DMF in the P2VP brushes. ${ }^{27,28}$ Further increase of the DMF fraction in the mixture gradually increased $\lambda_{2}^{\text {peak }}$ due to the reduced solubility of PS in the solvent. These results confirm the one-to-one matching between the solvent mixing ratio and the coordinate of $\left(\lambda_{1}^{\text {peak }}, \lambda_{2}^{\text {peak }}\right)$. Another example of the gradual change of $\lambda_{1}^{\text {peak }}$ and $\lambda_{2}^{\text {peak }}$ as a function of the mixing ratio of acetone and cyclohexane is demonstrated in Fig. 5b. In this case, the graph follows a U-shaped line because the solubility parameter $\left(\delta=16.8 \mathrm{MPa}^{1 / 2}\right)$ of cyclohexane is

(a)

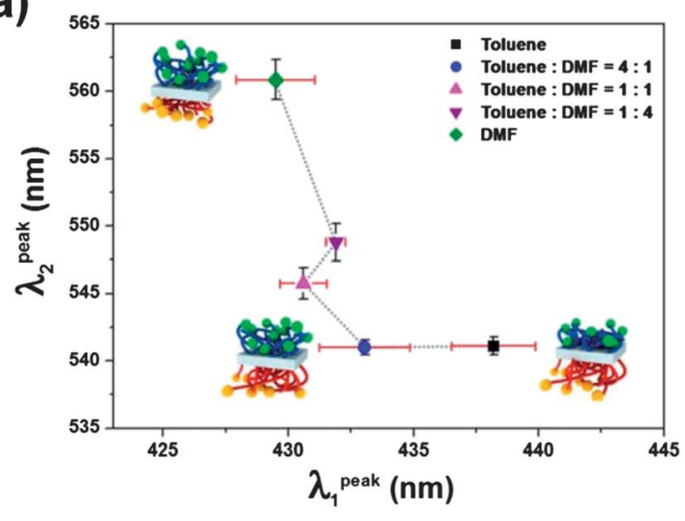

(b)

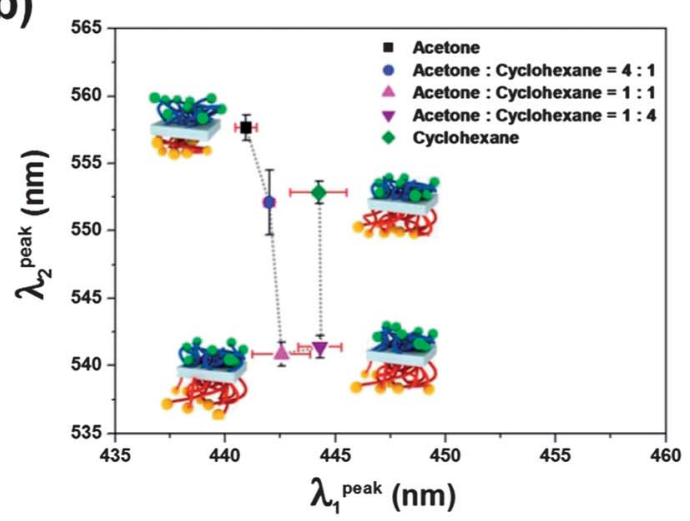

Fig. 5 Two-dimensional representation of the peak positions of $\left(\lambda_{1}^{\text {peak }}, \lambda_{2}^{\text {peak }}\right)$ for different mixing ratios of two miscible liquids. Mixtures of (a) toluene and DMF and (b) acetone and cyclohexane. The swelling and deswelling of the LSPR nanocomposites are schematically shown together with the data points. The peak positions are matched one to one with the mixing ratios. The data points are the averages of 10 measurements and the error bars are standard deviations. 
much lower than that of PS, and consequently, the solvent swells neither PS nor P2VP. With addition of acetone with a solubility parameter of $19.7 \mathrm{MPa}^{1 / 2}$ to cyclohexane, ${ }^{24}$ the effective solubility parameter of the mixed solvent approaches that of PS, resulting in a blue shift of $\lambda_{2}^{\text {peak }}$. Further increase of the acetone fraction (acetone-cyclohexane $=1: 1$ ) in the mixture makes P2VP also highly soluble in the solvent and, as a result, decreases $\lambda_{1}^{\text {peak }}$. However, greater addition of acetone significantly decreases the solubility of PS in the mixture solvent, causing a red shift of $\lambda_{2}^{\text {peak }}$. These two test results suggest that the dual-responsive nanocomposite sensor is also practical and efficient to estimate the mixing ratio of various solvents. In particular, this function will be very useful for the in situ monitoring of the change in solvent compositions in the production lines of electronics and chemical industries.

\section{Methods}

\section{Materials}

Poly(glycidyl methacrylate) $\left(M_{\mathrm{n}}=20 \mathrm{~kg} \mathrm{~mol}^{-1}\right)$, gold(III) chloride trihydrate $\left(\mathrm{HAuCl}_{4} \cdot 3 \mathrm{H}_{2} \mathrm{O}\right)$, tetraoctylammonium bromide (TOAB), 11-mercaptoundecanoic acid (MUA), silver nitrate $\left(\mathrm{AgNO}_{3}\right)$, and sodium borohydride $\left(\mathrm{NaBH}_{4}\right)$ were purchased from Sigma-Aldrich. Hydroxy-terminated poly(2-vinylpyridine) $\left(M_{\mathrm{n}}=13 \mathrm{~kg} \mathrm{~mol}^{-1}\right)$ and $\alpha$-hydroxy, $\omega$-carboxy-terminated polystyrene $\left(M_{\mathrm{n}}=9.5 \mathrm{~kg} \mathrm{~mol}{ }^{-1}\right)$ were purchased from Polymer Source Inc. Methanol, ethanol, $N, N$-dimethylformamide (DMF), pyridine, acetic acid, dichloromethane (DCM), acetone, tetrahydrofuran (THF), toluene, xylene, cyclohexane, heptane, and isopropyl alcohol (IPA) were purchased from Junsei Chemical Co., Ltd. The above chemicals were used as received. Deionized water (DI water) (resistivity $=18 \mathrm{M} \Omega \mathrm{cm}$ ), slide glasses (Marienfeld), and gel pads (Electron Microscopy Sciences) were also used.

\section{Fabrication of dual-responsive nanocomposites}

The glass substrates were cut into $1 \times 2 \mathrm{~cm}^{2}$ pieces and cleaned in acetone, IPA, and DI water with sonication for 10 minutes. The P2VP thin films were spin-coated from a pyridine solution ( $2 \mathrm{wt} \%$ ) onto a glass piece and then annealed at $180{ }^{\circ} \mathrm{C}$ for 18 hours. A PGMA thin layer was spin-coated onto the opposite side of the substrates using gel pads to prevent mechanical damage and chemical contamination by the spin-coating process. After 10 minutes of thermal annealing at $110{ }^{\circ} \mathrm{C}$, $\alpha$-hydroxy, $\omega$-carboxy-terminated thin PS brushes were anchored onto the PGMA thin films by spin-coating with a toluene solution ( $2 \mathrm{wt} \%$ ) followed by annealing at $150{ }^{\circ} \mathrm{C}$ for 3 hours. The sample was then cleaned with solvents (pyridine for P2VP and toluene for PS) several times to remove unreacted polymers. For the formation of silver nanoparticles, the sample was dipped in a $0.1 \mathrm{M}$ aqueous $\mathrm{AgNO}_{3}$ solution for 3 hours and cleaned with DI water. Immediately after the cleaning step, the sample was placed in an aqueous $0.2 \mathrm{M} \mathrm{NaBH}_{4}$ solution for 1 hour while stirring, which was followed by washing with DI water. This process was repeated three times. For the immobilization of gold nanoparticles on PS brushes, gold nanoparticles were prepared using a previously reported synthesis method. ${ }^{21,22}$ Au NPs were attached onto PS brushes by the drop-casting of a toluene solution several times and by rinsing with toluene.

\section{Microscopy imaging and optical measurement}

The surface topographic information of the nanocomposites was obtained using an atomic force microscope (Park systems, XE-100). Plane-view transmission electron microscopy (TEM) specimens for the observation of the Ag NPs were prepared by mechanical polishing followed by ion-milling the nanocomposites containing the NPs. The Au NP thin films were prepared via drop-casting the NPs dispersed in toluene and drying on a carbon-coated copper grid. A high-resolution TEM (FE-TEM, JEOL-2100F HR $200 \mathrm{kV}$ ) device was used. The absorption spectra were measured using a UV-Vis-NIR spectrophotometer (Shimadzu UV-3101 PC) equipped with a tungsten lamp in the wavelength range of $350 \mathrm{~nm}$ to $800 \mathrm{~nm}$. Quartz cuvettes filled with the respective organic liquids were used as reference samples.

\section{Conclusions}

We introduced a simple, fast, and accurate method to identify unknown liquids through the synergic combination of plasmonic nanocomposites with highly tunable and responsive absorption spectra. The nanocomposites, fabricated by attaching $\mathrm{Au}$ and $\mathrm{Ag}$ NPs onto hydrophobic and hydrophilic polymer brushes, respond differently against diverse solvents depending on the degree of interaction between the polymer brush and the surrounding solvent. We showed that the limited selectivity for the case of a single nanocomposite sensor can be effectively resolved when using the dual-responsive nanocomposite sensor by significantly reducing the overlap ranges between the absorption peaks. Furthermore, the compositions of mixed solvents can be estimated by the LSPR sensor because the coordinates of the two absorption peaks are matched one-toone to their mixing ratios. We expect that the LSPR sensor based on multiple nanocomposites with mutually complementary responsiveness to surrounding environments will be utilized for the collection of more diverse physical, chemical, and biological information.

\section{Acknowledgements}

This work was supported by the Center for Integrated Smart Sensors funded by the Ministry of Science, ICT \& Future Planning as Global Frontier Project (CISS-2012366054188).

\section{Notes and references}

1 H. A. Atwater and A. Polman, Nat. Mater., 2010, 9, 205-213.

2 H. Liu, B. Wang, L. Ke, J. Deng, C. C. Chum, S. L. Teo, L. Shen, S. A. Maier and J. H. Teng, Nano Lett., 2012, 12, 1549-1554.

3 S. Underwood and P. Mulvaney, Langmuir, 1994, 10, 34273430 .

4 P. Mulvaney, Langmuir, 1996, 12, 788-800. 
5 W. Rechberger, A. Hohenau, A. Leitner, J. R. Krenn, B. Lamprecht and F. R. Aussenegg, Opt. Commun., 2003, 220, 137-141.

6 E. Hutter and J. H. Fendler, Adv. Mater., 2004, 16, 1685-1706.

7 S. Szunerits and R. Boukherroub, Chem. Commun., 2012, 48, 8999-9010.

8 K. M. Mayer and J. H. Hafner, Chem. Rev., 2011, 111, 3828-3857.

9 A. W. Clark and J. M. Cooper, Angew. Chem., Int. Ed., 2012, 51, 3562-3566.

10 O. Kedem, A. B. Tesler, A. Vaskevich and I. Rubinstein, ACS Nano, 2011, 5, 748-760.

11 S. Lee and V. H. Perez-Luna, Langmuir, 2007, 23, 5097-5099.

12 S. Gupta, M. Agrawal, P. Uhlmann, F. Simon, U. Oertel and M. Stamm, Macromolecules, 2008, 41, 8152-8158.

13 I. Tokareva, S. Minko, J. H. Fendler and E. Hutter, J. Am. Chem. Soc., 2004, 126, 15950-15951.

14 M. P. Weir and A. J. Parnell, Polymer, 2011, 3, 2107-2132.

15 M. A. C. Stuart, W. T. S. Huck, J. Genzer, M. Muller, C. Ober, M. Stamm, G. B. Sukhorukov, I. Szleifer, V. V. Tsukruk, M. Urban, F. Winnik, S. Zauscher, I. Luzinov and S. Minko, Nat. Mater., 2010, 9, 101-113.

16 K. J. Albert, N. S. Lewis, C. L. Schauer, G. A. Sotzing, S. E. Stitzel, T. P. Vaid and D. R. Walt, Chem. Rev., 2000, 100, 2595-2626.
17 A. Hierlemann and R. Gutierrez-Osuna, Chem. Rev., 2008, 108, 563-613.

18 K. S. Iyer, B. Zdyrko, H. Malz, J. Pionteck and I. Luzinov, Macromolecules, 2003, 36, 6519-6526.

19 I. Luzinov, K. S. Iyer, V. Klep, B. Zdyrko, J. Draper and Y. Liu, Abstr. Pap. Am. Chem. Soc., 2003, 225, U641-U642.

20 J. Chai, D. Wang, X. N. Fan and J. M. Buriak, Nat. Nanotechnol., 2007, 2, 500-506.

21 J. Matsui, K. Akamatsu, S. Nishiguchi, D. Miyoshi, H. Nawafune, K. Tamaki and N. Sugimoto, Anal. Chem., 2004, 76, 1310-1315.

22 K. H. Kim, M. Yamada, D. W. Park and M. Miyake, Chem. Lett., 2004, 344-345.

23 S. Gupta, P. Uhlmann, M. Agrawal, S. Chapuis, U. Oertel and M. Stamm, Macromolecules, 2008, 41, 2874-2879.

24 A. F. M. Barton, CRC handbook of solubility parameters and other cohesion parameters, CRC Press, Boca Raton, 1991.

25 L. Cui, H. F. Wang, Y. Ding and Y. C. Han, Polymer, 2004, 45, 8139-8146.

26 S. Link, M. B. Mohamed and M. A. El-Sayed,J. Phys. Chem. B, 1999, 103, 3073-3077.

27 P. Auroy and L. Auvray, Macromolecules, 1992, 25, 41344141.

28 P. Auroy and L. Auvray, Langmuir, 1994, 10, 225-231. 